\title{
An Upwind Finite Volume Element Method for Nonlinear Convection Diffusion Problem
}

\author{
Fuzheng Gao, Yirang Yuan, Ning Du \\ School of Mathematics, Shandong University, Jinan, China \\ E-mail:fzgao@sdu.edu.cn \\ Received August 17, 2011; revised September 6, 2011; accepted September 15, 2011
}

\begin{abstract}
A class of upwind finite volume element method based on tetrahedron partition is put forward for a nonlinear convection diffusion problem. Some techniques, such as calculus of variations, commutating operators and the a priori estimate, are adopted. The a priori error estimate in $L^{2}$-norm and $H^{1}$-norm is derived to determine the error between the approximate solution and the true solution.
\end{abstract}

Keywords: Nonlinear, Convection-Diffusion, Tetrahedron Partition, Error Estimates

\section{Introduction}

Consider the following nonlinear convection-diffusion problem:

$$
\begin{gathered}
u_{t}-\mu \Delta u+\nabla \cdot \mathbf{F}(\mathbf{x}, u)=g(\mathbf{x}, u), \quad \mathbf{x} \in \Omega, t \in J \\
u(\mathbf{x}, t)=0, \quad \mathbf{x} \in \Gamma, t \in J, \\
u(\mathbf{x}, 0)=u_{0}(\mathbf{x}), \quad \mathbf{x} \in \Omega,
\end{gathered}
$$

where $\Omega \subset R^{3}$ is a bounded region with piecewise smooth boundary $\Gamma . \mu$ is a small positive constant and $\mathbf{F}(\mathbf{x}, u)=\left(f_{1}(\mathbf{x}, u), f_{2}(\mathbf{x}, u), f_{3}(\mathbf{x}, u)\right)$ is a smooth vector function on $\bar{\Omega} \times R, \mathbf{F}(\mathbf{x}, 0)=0$.

The finite volume element method (FVEM) is a discrete technique for partial differential equations, especially for those arising from physical conservation laws, including mass, momentum and energy. This method has been introduced and analyzed by R. Li and his collaborators since 1980s, see [1] for details. The FVEM uses a volume integral formulation of the original problem and a finite partitioning set of covolumes to discretize the equations. The approximate solution is chosen out of a finite element spaces [1-3] The FVEM is widely used in computational fluid mechanics and heat transfer problems [2-5]. It possesses the important and crucial property of inheriting the physical conservation laws of the original problem locally. Thus it can be expected to capture shocks, or to study other physical phenomena more effectively.

On the other hand, the convection-dominated diffusion problem has strong hyperbolic characteristics, and there- fore the numerical method is very difficult in mathematics and mechanics. when the central difference method, though it has second-order accuracy, is used to solve the convection-dominated diffusion problem, it produces numerical diffusion and oscillation near the discontinuous domain, making numerical simulation failure. The case usually occurs when the finite element methods (FEM) and FVEM are used for solve the convectiondominated diffusion problem.

For the two-phase plane incompressible displacement problem which is assumed to be $\Omega$-periodic, J. Douglas, Jr., and T.F.Russell have published some articles on the characteristic finite difference method and FEM to solve the convection-dominated diffusion problems and to overcome oscillation and faults likely to occur in the traditional method [6]. Tabata and his collaborators have been studying upwind schemes based triangulation for convection-diffusion problem since 1977 [7-11]. Yuan, starting from the practical exploration and development of oil-gas resources, put forward the upwind finite difference fractional steps methods for the two-phase threedimensional compressible displacement problem [12].

Most of the papers known concern on the FVEM for one- and two-dimensional linear partial differential equations $[1-4,13,14]$. In recent years, M. Feistauer [15,16], by introducing lumping operator, constructed finite volume-finite element method for nonlinear convectiondiffusion problems. On the other hand, because the FEM costs great expense to solve the three-dimensional problems, finite difference methods (FDM) are usually used to approximate the problems [12]. These works inspire 
us to look into the subject how to use the upwind FVEM to solve three-dimensional nonlinear convection-dominated diffusion problems. In this article, we continue to our work [17] and put forward an upwind FVEM for three-dimensional nonlinear convection-dominated diffusion problems based on tetrahedron partition and its dual partition of $\Omega$. Some techniques, such as calculus of variations, commutating operator and the a priori error estimate, are adopted. The a priori error estimate in $L^{2}$-norm and $H^{1}$-norm is derived to determine the error between the approximate solution and the true solution.

The remainder of this paper is organized as follows. In Section 2, we put forward the upwind FVEM for problem (1). In this section, we introduce notations, construct tetrahedron mesh partition $T_{h}$ of $\Omega$ and its dual partition. Some auxiliary lemmas and the a priori error estimate in $L^{2}$-norm and $H^{1}$-norm of the scheme are shown In Section 3 and Section 4, respectively. In Section 5, some concluding remarks are presented.

Throughout this paper we use $C$ (without or with subscript) to denote a generic constant independent of discrete parameters. We also adopt the standard notations of Sobolev spaces and norms and semi-norms as in $[18,19]$.

\section{The UFVE Method}

Suppose problem (1) satisfy condition (A1):

$\left(C_{1}\right)$ Continuity condition: $g(\mathbf{x}, u) \in L^{2}(\Omega \times R)$ is Lipschitz continuous w.r.t. the second variable $u$.

$\left(C_{2}\right)$ The vector function $\mathbf{F}(\mathbf{x}, u)$ has 1-order continuous partial derivative w.r.t. $\mathbf{x}$ and $u$.

Suppose the true solution of problem (1) possess certain smooth and satisfy:

(R) Regular condition:

$$
u \in W^{2, \infty}\left(L^{\infty}\right) \cap H^{1}\left(L^{2}\right) \cap L^{\infty}\left(H^{2}\right) .
$$

Before presenting the numerical scheme we introduce some notations. For simplicity we assume $\Omega$ is the domain $\Omega=\left(0, X_{L}\right) \times\left(0, Y_{L}\right) \times\left(0, Z_{L}\right)$. Firstly, Let us consider a family of regular tetrahedron partition $T_{h}$ in the domain $\bar{\Omega}$, which is a closure of $\Omega$. Let $h$ be maximum diameter of cell of $T_{h}$. For a fixed tetrahedron partition $T_{h}=\{K\}$, we define a closed tetrahedron set $\left\{K_{i}\right\}_{i=1}^{N_{K}}$ and node set

$$
\bar{\Omega}_{h}=\Omega_{0} \bigcup \Gamma_{h}=\left\{P_{i}\right\}_{i=1}^{M_{1}} \bigcup\left\{P_{i}\right\}_{i=M_{1}+1}^{M_{2}}=\left\{P_{i}\right\}_{i=1}^{M_{2}},
$$

where $\Omega_{0}$ is inner nodes set of $\Omega$ and $\Gamma_{h}$ boundary nodes set on $\Gamma$. Let $E_{h}=\left\{e_{i}: 1 \leq i \leq M_{E}\right\}$ be all edges set.

Definition 2.1. Suppose that $T=\left\{T_{h}: 0<h \leq h_{0}\right\}$ is a set of tetrahedron partition of $\Omega$, the set $T_{h}$ is called regular, if there exists a positive constant $\sigma_{1}$ independent of $h$, such that

$$
\max _{K \in T_{h}} h_{K} \rho_{K} \leq \sigma_{1}, \forall h \in\left\{0, h_{0}\right\}
$$

where $h_{K}$ and $\rho_{K}$ are the diameter of $K$ and the maximum diameter of circumscribing sphere of tetrahedron $K$, respectively.

Definition 2.2. The two tetrahedron cells are called faceadjacent if they have one common face, while edge-adjacent if they have one common edge.

Definition 2.3. The two nodes are called adjacent if they form one edge which belongs to $E_{h}$. Denote by $\Lambda_{i}=\left\{j: P_{j}\right.$ is adjacent to $\left.P_{i}, P_{i}, P_{j} \in \Omega_{h}\right\}$.

For a given tetrahedron partition $T_{h}$ with nodes $\left\{P_{i}\right\} \in \Omega_{h}$ and edges $\left\{e_{i}\right\} \in E_{h}$, we construct two kind of dual partitions. First, we will construct the circumcenter dual partition of $T_{h} . \forall P_{i} \in \Omega_{h}$, let

$\Omega_{h}\left(P_{i}\right)=\left\{K: K \in T_{h}, P_{i}\right.$ is a vertex of $\left.K\right\}$. Let $Q_{j}$ be a circumcenter of $K\left(\in \Omega_{h}\left(P_{i}\right)\right)$. Connecting $Q_{j}$ of the two face-adjacent tetrahedron cells which belong to $\Omega_{h}\left(P_{i}\right)$, then we can derive a polyhedron $K_{P_{i}}^{*}$ which surrounds the node $P_{i} . Q_{j}$ are vertices of the polyhedron $K_{P_{i}}^{*}$ which is called circumcenter dual partition corresponding to node $P_{i} . T_{h}^{*}=\left\{K_{P_{i}}^{*}: P_{i} \in \Omega_{h}\right\}$ is the circumcenter dual partition of $T_{h}$. Denote by $P_{i j}$ the midpoint of $P_{i}$ and its adjacent node $P_{j}$.

The other dual partition as follows. $\forall e_{k} \in E_{h}$, let $\Omega_{h}\left(e_{k}\right)=\left\{K: K \in T_{h}\right.$ and $e_{k}$ is a edge of $\left.K\right\}$. Denote by $P_{k_{1}}$ and $P_{k_{2}}$ the vertices of the edge $e_{k}$ and $Q_{j}$ the circumcenter of the $K \in \Omega_{h}\left(e_{k}\right)$. Suppose $K_{e_{k}}^{*}$ is a polyhedron whose vertices are $P_{k_{1}}, P_{k_{2}}, Q_{j} s . K_{e_{k}}^{*}$ is called dual cell for edge $e_{k} \cdot \bar{T}_{h}^{*}=\left(K_{e_{k}}^{*}\right)_{k=1}^{M_{E}}$ is the other dual partition to $T_{h}$.

Let $\Omega_{h}^{*}$ be the node set of dual partition. For $Q \in \Omega_{h}^{*}$, let $K_{Q}$ be tetrahedron cell which includes $Q$. Let $\left|K_{P}^{*}\right|$ and $\left|K_{Q}\right|$ be volumes of dual cell $K_{P}^{*}$ and tetrahedron cell $K_{Q}$, respectively. Let $h$ be the diameter of tetrahedron cell $K_{Q}$. As follows, we assume that the partition family $T_{h}$ is regular, i.e., there exist positive constants $C_{1}, C_{2}$ independent of $h$, such that the following condition (A2) satisfies:

$$
\begin{cases}C_{1} h^{3} \leq\left|K_{p}^{*}\right| \leq C_{2} h^{3}, & P \in \bar{\Omega}_{h}, \\ C_{1} h^{3} \leq\left|K_{Q}\right| \leq C_{2} h^{3}, & Q \in \Omega_{h}^{*} .\end{cases}
$$

Suppose that a trial function space $U_{h} \subset H_{0}^{1}(\Omega)$, whose basis functions are $\left\{\phi\left(P_{i}\right)\right\}_{i=1}^{M_{1}}$ possessing the form $\left(\alpha_{0}+\alpha_{1} x+\alpha_{2} y+\alpha_{3} z\right)$ based on $T_{h}$ [15], and $\phi\left(P_{i}\right)=0, P_{i} \in \Gamma_{h}$. Test function space $V_{h} \subset L^{2}(\Omega)$ is a piecewise constant function space corresponding to the dual partition $T_{h}^{*}$, whose basis functions are $\left\{\psi(P), P \in \Omega_{h}\right\}$. 


$$
\psi(P)=\left\{\begin{array}{lc}
1, & P \in K_{P}^{*}, \\
0, & \text { otherwise, }
\end{array}\right.
$$

and $\psi(P)=0, P \in \Gamma_{h}$.

For the following analysis, we introduce two interpolation operators. Suppose that $\Pi_{h}$ and $\Pi_{h}^{*}$ are interpolation operators from $H_{0}^{1}$ to $U_{h}$ and $V_{h}$, respectively, satisfying

$$
\begin{gathered}
\Pi_{h} u=\sum_{i=1}^{M_{1}} u\left(P_{i}\right) \phi\left(P_{i}\right) . \\
\Pi_{h}^{*} u=\sum_{K_{P}^{*} \in T_{h}^{*}} u(P) \psi(P) .
\end{gathered}
$$

Multiplying both sides of (1) by $v$, integrating on dual partition cell $K_{P_{i}}^{*}$, using Green formula, and summing with respect to $P_{i} \in \Omega_{h}$, we have

$$
\left(u_{t}, v\right)+a(u, v)+b(u, v)=(g, v), v \in H_{0}^{1}(\Omega),
$$

where

$$
\begin{aligned}
& a(u, v)=\sum_{P_{i} \in \Omega_{h}}\left[\mu \int_{K_{P_{i}}^{*}} \nabla u \cdot \nabla v \mathrm{~d} \mathbf{x}-\mu \int_{\partial K_{P_{i}}^{*}} \partial u \partial v v \mathrm{~d} s\right], \\
& b(u, v)=-\sum_{P_{i} \in \Omega_{h}}\left(\int_{K_{P_{i}}^{*}} \mathbf{F} \cdot \nabla v \mathrm{~d} \mathbf{x}-\int_{\left.\partial K_{P_{i}}^{*} \mathbf{F} \cdot v v \mathrm{~d} s\right) .}\right.
\end{aligned}
$$

Converting $\mathbf{F}$ into [1]

$$
\mathbf{F}(\mathbf{x}, u)=\int_{0}^{u} \partial \mathbf{F}(\mathbf{x}, \bar{u}) \partial \bar{u} \mathrm{~d} \bar{u} .
$$

Let

$$
\begin{aligned}
& \beta_{i j}^{+}(\mathbf{x}, u)=\int_{0}^{u} \max \left(0, \partial \mathbf{F}(\mathbf{x}, \bar{u}) \partial \bar{u} \cdot v_{i j}\right) \mathrm{d} \bar{u}, \\
& \beta_{i j}^{-}(\mathbf{x}, u)=\int_{0}^{u} \max \left(0,-\partial \mathbf{F}(\mathbf{x}, \bar{u}) \partial \bar{u} \cdot v_{i j}\right) \mathrm{d} \bar{u},
\end{aligned}
$$

where $v_{i j}$ is the unit outward normal vector of $\Gamma_{i j} \subset \partial K_{P_{i}}^{*}$. For $u_{h} \in U_{h}, v_{h} \in V_{h}$, we introduce bilinear form

$$
\begin{aligned}
& b_{h}\left(u_{h}, v_{h}\right)=\sum_{P_{i} \in \Omega_{h}} v_{h}\left(P_{i}\right) \sum_{j \in \Lambda_{i}}\left|\Gamma_{i j}\right| \\
& \cdot\left[\beta_{i j}^{+}\left(P_{i j}, u_{h}\left(P_{i}\right)\right)-\beta_{i j}^{-}\left(P_{i j}, u_{h}\left(P_{j}\right)\right)\right],
\end{aligned}
$$

where $\left|\Gamma_{i j}\right|$ is the area of $\Gamma_{i j}$.

So far, we can obtain the semi-discrete upwind finite volume element scheme: Find $u_{h} \in U_{h}$, such that

$$
\left(u_{h, t}, v_{h}\right)+a\left(u_{h}, v_{h}\right)+b_{h}\left(u_{h}, v_{h}\right)=\left(g\left(\mathbf{x}, u_{h}\right), v_{h}\right) \text {, }
$$

where

$$
a\left(u_{h}, v_{h}\right)=-\sum_{P_{i} \in \Omega_{h}} \mu \int_{\partial K_{P_{l}^{*}}^{*}} \partial u_{h} \partial v v_{h} \mathrm{~d} s .
$$

Let $\Delta t=T / N$, denote by $t^{n}=n \Delta t, u^{n}=u\left(t^{n}\right)$,

$$
u_{h}^{n}=u_{h}\left(t^{n}\right), n=1,2, \cdots, N, \partial_{t} u_{h}^{n-1}=\left(u_{h}^{n}-u_{h}^{n-1}\right) / \Delta t .
$$

If approximate solution $u_{h}^{n-1} \in U_{h}$ is known, then $u_{h}^{n}$ can be found by the following full-discrete upwind finite volume element scheme.

$$
\begin{aligned}
& \left(\Pi_{h}^{*} \partial_{t} u_{h}^{n-1}, v_{h}\right)+a\left(u_{h}^{n-1}, v_{h}\right)+b_{h}\left(u_{h}^{n-1}, v_{h}\right) \\
& =\left(g\left(\mathbf{x}, u_{h}^{n-1}\right), v_{h}\right) .
\end{aligned}
$$

\section{Auxiliary Lemmas}

Define the discrete norm and the discrete semi-norm [1] as follows.

$$
\begin{gathered}
\left\|u_{h}\right\|_{0, h}^{2}=\left\|\Pi_{h}^{*} u_{h}\right\|_{0}^{2}=\sum_{K_{P_{i}}^{*} \in T_{h}^{*}}\left(u_{h}\left(P_{i}\right)\right)^{2}\left|K_{P_{i}}^{*}\right|, \\
\left|u_{h}\right|_{1, h}^{2}=\sum_{k=1}^{M_{E}}\left(u_{h}\left(P_{k 2}\right)-u_{h}\left(P_{k 1}\right)\left|e_{k}\right|\right)^{2}\left|K_{e_{k}}^{*}\right|, \\
\left\|u_{h}\right\|_{1, h}^{2}=\left\|u_{h}\right\|_{0, h}^{2}+\left|u_{h}\right|_{1, h}^{2},
\end{gathered}
$$

obviously, the discrete norm and the discrete semi-norm are equivalent to the continuous norm and the full-norm on $U_{h}$, respectively.

Lemma 1. Suppose all cells $K_{Q}$ of the partition $T_{h}$ satisfy conditions (A2), $T_{h}^{*}$ is a circumcenter dual partition. $\forall u_{h}, u_{h} \in U_{h}$, there exist positive constants $\gamma, C_{0}$ independent of $h$, such that

$$
\begin{gathered}
a\left(u_{h}, \Pi_{h}^{*} u_{h}\right) \geq \gamma\left\|u_{h}\right\|_{1}^{2}, \quad \forall u_{h} \in U_{h} . \\
a\left(u_{h}, \Pi_{h}^{*} \bar{u}_{h}\right) \leq C_{0}\left\|u_{h}\right\|_{1}\left\|\bar{u}_{h}\right\|_{1}, \forall u_{h}, \bar{u}_{h} \in U_{h}, \\
a\left(u_{h}, \Pi_{h}^{*} u_{h}\right)=a\left(u_{h}, \Pi_{h}^{*} u_{h}\right), \quad \forall u_{h}, \bar{u}_{h} \in U_{h} .
\end{gathered}
$$

Remarks:

1) From Lemma 1, we know that $a(\cdot, \cdot)$ is symmetrical and positive definite in $U_{h}$.

2) Let $\left\|u_{h}\right\|_{1}=\left[a\left(u_{h}, \Pi_{h}^{*} u_{h}\right)\right]^{\frac{1}{2}}$, then $\|\cdot\| \|_{1}$ is equivalent to $|\cdot|_{1}$ in $U_{h}$.

Lemma 2. Let $\left\|u_{h}\right\|_{0}=\left(\Pi_{h}^{*} u_{h}, \Pi_{h}^{*} u_{h}\right)^{\frac{1}{2}},\|\cdot\| \|_{0}$ is equivalent to $\|\cdot\|_{0}$ in $U_{h}$.

The proof of lemma 2 can be completed by computing integral on cell $K_{Q}$, directly.

Theorem 1. (Trace Theorem) [20]. Suppose that $\Omega$ has a piecewise Lipschitz boundary, and that $p$ is a real number in range $1 \leq p \leq \infty$. Then there exists a constant $C$, such that

$$
\|v\|_{L^{p}(\partial \Omega)} \leq C\|v\|_{L^{p}(\Omega)}^{1-1 / p}\|v\|_{W_{p}^{1}(\Omega)}^{1 / p}, \quad \forall v \in W_{p}^{1}(\Omega) .
$$

Lemma 3. For $h$ small enough, suppose $P^{\prime}$ is a random point in dual partition cell $K_{P_{i}}^{*}$,

$$
\Gamma_{i j}=K_{P_{i}}^{*} \bigcap K_{P_{j}}^{*}
$$


then

$$
\sum_{j \in \Lambda_{i}} \iint_{\Gamma_{i j}}\left|u\left(P^{\prime}\right)-u(\mathbf{x})\right| \mathrm{d} s \leq C h^{2}\left(\|u\|_{1, K_{P_{i}}^{*}}+\|u\|_{2, K_{P_{i}}^{*}}\right) .
$$

Proof. From Hölder inequality, we can get that

$$
\begin{aligned}
& \sum_{j \in \Lambda_{i}} \iint_{\Gamma_{i j}}\left|u\left(P^{\prime}\right)-u(\mathbf{x})\right| \mathrm{d} s \\
& \leq C h \sum_{j \in \Lambda_{i}}\left(\iint_{\Gamma_{i j}}\left|u\left(P^{\prime}\right)-u(\mathbf{x})\right|^{2} \mathrm{~d} s\right)^{\frac{1}{2}}
\end{aligned}
$$

Using Taylor expansion, trace theory in which we choose $p=2$ and Hölder inequality, we can complete the proof of lemma 3 .

Lemma 4. For $\forall u_{h}, \bar{u}_{h} \in U_{h}$, there exists a positive constant $C$, such that

$$
\begin{gathered}
\left(u_{h}, \Pi_{h}^{*} \bar{u}_{h}\right)=\left(u_{h}, \Pi_{h}^{*} \bar{u}_{h}\right), \\
\left(u_{h}, \Pi_{h}^{*} \bar{u}_{h}\right) \leq C\left\|u_{h}\right\|_{0} \cdot\left\|\bar{u}_{h}\right\|_{0} .
\end{gathered}
$$

Proof. From the properties of the functions in $U_{h}$, for each partition cell $K\left(\in T_{h}\right)$, we know that $\left.u_{h}\right|_{K}$ has the following expression.

$$
\begin{aligned}
& \left.u_{h}(x, y, z, t)\right|_{K}=u\left(x_{i_{0}}, y_{i_{0}}, z_{i_{0}}, t\right) \lambda_{i_{0}}+u\left(x_{i_{1}}, y_{i_{1}}, z_{i_{1}}, t\right) \lambda_{i_{1}} \\
& +u\left(x_{i_{2}}, y_{i_{2}}, z_{i_{2}}, t\right) \lambda_{i_{2}}+u\left(x_{i_{3}}, y_{i_{3}}, z_{i_{3}}, t\right) \lambda_{i_{3}},
\end{aligned}
$$

where

$$
\lambda_{l}=\frac{1}{6 V e}\left(a_{l} x+b_{l} y+c_{l} z+d_{l}\right), l=i_{0}, i_{1}, i_{2}, i_{3},
$$

and $V e$ is the volume of tetrahedron $P_{i_{0}}-P_{i_{1}} P_{i_{2}} P_{i_{3}}$, i.e.,

$$
V e=\frac{1}{6}\left|\begin{array}{cccc}
1 & x_{i_{0}} & y_{i_{0}} & z_{i_{0}} \\
1 & x_{i_{1}} & y_{i_{1}} & z_{i_{1}} \\
1 & x_{i_{2}} & y_{i_{2}} & z_{i_{2}} \\
1 & x_{i_{3}} & y_{i_{3}} & z_{i_{3}}
\end{array}\right| .
$$

$P_{i_{l}}\left(l=i_{0}, i_{1}, i_{2}, i_{3}\right)$, whose coordinates are $\left(x_{i_{l}}, y_{i_{l}}, z_{i_{l}}\right)$, are four vertices of tetrahedron cell $P_{i_{0}}-P_{i_{1}} P_{i_{2}} P_{i_{3}}$ which belongs to $T_{h} . \lambda_{l}\left(l=i_{0}, i_{1}, i_{2}, i_{3}\right)$ are the volume coordinates which are corresponding to tetrahedron cell $P_{i_{0}}-P_{i_{1}} P_{i_{2}} P_{i_{3}}$. For $l=i_{0}$,

$$
\begin{aligned}
& a_{i_{0}}=-\left|\begin{array}{lll}
1 & y_{i_{1}} & z_{i_{1}} \\
1 & y_{i_{2}} & z_{i_{2}} \\
1 & y_{i_{3}} & z_{i_{3}}
\end{array}\right|, b_{i_{0}}=\left|\begin{array}{lll}
1 & x_{i_{1}} & z_{i_{1}} \\
1 & x_{i_{2}} & z_{i_{2}} \\
1 & x_{i_{3}} & z_{i_{3}}
\end{array}\right|, \\
& c_{i_{0}}=-\left|\begin{array}{lll}
1 & x_{i_{1}} & y_{i_{1}} \\
1 & x_{i_{2}} & y_{i_{2}} \\
1 & x_{i_{3}} & y_{i_{3}}
\end{array}\right|, d_{i_{0}}=\left|\begin{array}{lll}
x_{i_{1}} & y_{i_{1}} & z_{i_{1}} \\
x_{i_{2}} & y_{i_{2}} & z_{i_{2}} \\
x_{i_{3}} & y_{i_{3}} & z_{i_{3}}
\end{array}\right| .
\end{aligned}
$$

Analogously, we can define the remaining coefficients $a_{l}, b_{l}, c_{l}, d_{l}\left(l=i_{1}, i_{2}, i_{3}\right)$. Further,

$$
\left(u_{h}, \Pi_{h}^{*} u_{h}\right)=\sum_{K \in T_{h}} \sum_{l=i_{0}, i_{1}, i_{2}, i_{3}} u_{h}\left(P_{l}\right) \iiint_{K_{P_{l}}^{*} \cap K} u_{h} \mathrm{~d} x \mathrm{~d} y \mathrm{~d} z .
$$

For simplifying numerical integral, we divide the polyhedron integral domain $K_{P_{10}}^{*} \cap K$ into six tetrahedron integral domains

$$
\begin{aligned}
& V_{1}=\text { tetrahedron } P_{i_{0}}-P_{i_{0} i_{1}} P_{i_{0_{1}} i_{2}} P_{i_{0} i_{1} i_{2} i_{3}}, \\
& V_{2}=\text { tetrahedron } P_{i_{0}}-P_{i_{0} i_{1}} P_{i_{0} i_{1} i_{3}} P_{i_{0} i_{1} i_{3} i_{3}} \text {, } \\
& V_{3}=\text { tetrahedron } P_{i_{0}}-P_{i_{0} i_{2}} P_{i_{0} i_{1} i_{2}} P_{i_{0} i_{1} i_{2} i_{3}} \text {, } \\
& V_{4}=\text { tetrahedron } P_{i_{0}}-P_{i_{0} i_{2}} P_{i_{0} i_{2} i_{3}} P_{i_{0} i_{1} i_{3} i_{3}} \text {, } \\
& V_{5}=\text { tetrahedron } P_{i_{0}}-P_{i_{0} i_{3}} P_{i_{0} i_{1} i_{3}} P_{i_{0} i_{1} i_{2} i_{3}} \text {, } \\
& V_{6}=\text { tetrahedron } P_{i_{0}}-P_{i_{0} i_{3}} P_{i_{0} i_{2} i_{3}} P_{i_{0} i_{1} i_{2} i_{3}} \text {, }
\end{aligned}
$$

where $P_{i_{0} i_{1}}$ is the midpoint of segment $P_{i_{0}} P_{i_{1}}$ while $P_{i_{0} i_{1} i_{2}}$ and $P_{i_{0} i_{2} i_{3}}$ are circumcenters of triangular surface $\triangle P_{i_{0}} P_{i_{1}} P_{i_{2}}$ and tetrahedron $P_{i_{0}}-P_{i_{1}} P_{i_{2}} P_{i_{3}}$, respectively. Analogously, we can define the remaining points.

Noting the Equality (24), we have that

$$
\begin{aligned}
\iiint_{K_{P_{1}}^{*} \cap K} u_{h} \mathrm{~d} x \mathrm{~d} y \mathrm{~d} z= & \left.\iiint_{K_{P_{1}}^{*} \cap K} u_{h}\right|_{K} \mathrm{~d} x \mathrm{~d} y \mathrm{~d} z \\
& =\iiint_{K_{P_{1}}^{*} \cap K}\left[u\left(P_{i_{0}}, t\right) \lambda_{i_{0}}+u\left(P_{i_{1}}, t\right) \lambda_{i_{1}}\right. \\
& \left.+u\left(P_{i_{2}}, t\right) \lambda_{i_{2}}+u\left(P_{i_{3}}, t\right) \lambda_{i_{3}}\right] \mathrm{d} x \mathrm{~d} y \mathrm{~d} z .
\end{aligned}
$$

For simplicity, we will omit the variable $t$ in function $u(x, y, z, t)$. From volume coordinate formula, noting $\lambda_{i_{0}}+\lambda_{i_{1}}+\lambda_{i_{2}}+\lambda_{i_{3}}=1$, we can derive

$$
\begin{aligned}
& \left.\iiint_{K_{P_{i_{0}}}^{*} \cap K} u_{h}\right|_{K} \mathrm{~d} x \mathrm{~d} y \mathrm{~d} z \\
& =\sum_{j=1}^{6} \iiint_{V_{j}}\left[u\left(P_{i_{0}}\right) \lambda_{i_{0}}+u\left(P_{i_{1}}\right) \lambda_{i_{1}}\right. \\
& \left.+u\left(P_{i_{2}}\right) \lambda_{i_{2}}+u\left(P_{i_{3}}\right) \lambda_{i_{3}}\right] \mathrm{d} x \mathrm{~d} y \mathrm{~d} z \\
& =\frac{|K|}{48}\left[-7 u\left(P_{i_{0}}\right)+3 u\left(P_{i_{1}}\right)+3 u\left(P_{i_{2}}\right)+3 u\left(P_{i_{3}}\right)\right]
\end{aligned}
$$

Further,

$$
\left(u_{h}, \Pi_{h}^{*} u_{h}\right)=\sum_{K \in T_{h}} \frac{|K|}{48} \alpha\left(\begin{array}{cccc}
-7 & 3 & 3 & 3 \\
3 & -7 & 3 & 3 \\
3 & 3 & -7 & 3 \\
3 & 3 & 3 & -7
\end{array}\right)
$$

where

$$
\alpha=\left(u_{h}\left(P_{i_{0}}\right), u_{h}\left(P_{i_{1}}\right), u_{h}\left(P_{i_{2}}\right), u_{h}\left(P_{i_{3}}\right)\right)
$$

and

$$
\beta=\left(u_{h}\left(P_{i_{0}}\right), u_{h}\left(P_{i_{1}}\right), u_{h}\left(P_{i_{2}}\right), u_{h}\left(P_{i_{3}}\right)\right)^{T} \cdot \beta,
$$

From the above equality, we can complete the proof of 
Lemma 4 easily.

\section{Convergence Analysis}

Now we consider the error estimates of the approximate solution. Let

$$
u^{n}-u_{h}^{n}=\left(u^{n}-\Pi_{h} u^{n}\right)+\left(\Pi_{h} u^{n}-u_{h}^{n}\right)=\rho_{h}^{n}+e_{h}^{n} .
$$

Choosing $t=t^{n-1}$ in (7), then we have

$$
\left(u_{t}\left(t^{n-1}\right), v\right)+a\left(u^{n-1}, v\right)+b\left(u^{n-1}, v\right)=\left(g\left(\mathbf{x}, u^{n-1}\right), v\right) .
$$

Subtracting (14) from (25), we obtain that

$$
\begin{aligned}
& \left(\Pi_{h}^{*} \partial_{t} e_{h}^{n-1}, v_{h}\right)+a\left(e_{h}^{n-1}, v_{h}\right) \\
& =\left(r^{n}, v_{h}\right)+a\left(-\rho_{h}^{n-1}, v_{h}\right) \\
& +\left(b_{h}\left(u_{h}^{n-1}, v_{h}\right)-b\left(u^{n-1}, v_{h}\right)\right) \\
& +\left(g\left(\mathbf{x}, u^{n-1}\right)-g\left(\mathbf{x}, u_{h}^{n-1}\right), v_{h}\right),
\end{aligned}
$$

where $r^{n}=\Pi_{h}^{*} \partial_{t} \Pi_{h} u^{n-1}-u_{t}\left(t^{n-1}\right)$.

Choosing $v_{h}=\Pi_{h}^{*}\left(e_{h}^{n-1}+e_{h}^{n}\right)$ in Equality (26), denote by $W_{1}, W_{2}$ and $T_{1}, T_{2}, T_{3}, T_{4}$ the left and right hand side terms of Equality (26), respectively. We will analyze the six terms successively.

For $W_{1}$, from the definition of $\|\cdot\| \|_{0}$, we have that

$$
W_{1}=\frac{1}{2 \Delta t}\left(\left\|e_{h}^{n}\right\|_{0}^{2}-\left\|e_{h}^{n-1}\right\|_{0}^{2}\right)
$$

Rewriting $W_{2}$ as

$$
\begin{aligned}
& W_{2}=a\left(e_{h}^{n-1}+e_{h}^{n}, \Pi_{h}^{*}\left(e_{h}^{n}+e_{h}^{n-1}\right)\right) \\
& +\left(a\left(e_{h}^{n-1}, \Pi_{h}^{*} e_{h}^{n-1}\right)-a\left(e_{h}^{n}, \Pi_{h}^{*} e_{h}^{n}\right)\right) \\
& +a\left(e_{h}^{n-1}, \Pi_{h}^{*} e_{h}^{n}\right)-a\left(e_{h}^{n}, \Pi_{h}^{*} e_{h}^{n-1}\right) \\
& =W_{21}+W_{22}+W_{23} .
\end{aligned}
$$

From (20) of Lemma 1, we can get the estimate to $W_{23}$ as follows.

$$
W_{23}=0 .
$$

From (27)-(29), we have

$$
\begin{aligned}
& W_{1}+W_{2} \geq \frac{1}{2 \Delta t}\left[\left(\left\|e_{h}^{n}\right\|_{0}^{2}-\frac{\Delta t}{2}\left\|e_{h}^{n}\right\|_{1}^{2}\right)\right. \\
& \left.-\left(\left\|e_{h}^{n-1}\right\|_{0}^{2}-\frac{\Delta t}{2}\left\|e_{h}^{n-1}\right\|_{1}^{2}\right)\right]+\frac{1}{4}\left\|e_{h}^{n-1}+e_{h}^{n}\right\|_{1}^{2}
\end{aligned}
$$

For each terms of the right hand side of (26). Using interpolation theory, triangulation inequality and lemma 4 , we know that

$$
\left|T_{1}\right| \leq C\left(\left\|e_{h}^{n-1}\right\|_{0}^{2}+\left\|e_{h}^{n}\right\|_{0}^{2}+(\Delta t)^{2}\left\|u_{t t}^{n-1}\right\|_{0}^{2}+h^{4}\left|u_{t}^{n-1}\right|_{2}^{2}\right) .
$$

Similarly, we can bound $T_{2}$ as

$$
\left|T_{2}\right| \leq C h\left|u^{n-1}\right|_{2} \cdot\left\|e_{h}^{n-1}+e_{h}^{n}\right\|_{1} .
$$

Further, making use of triangulation inequality and important inequality, we have that

$$
\left|T_{2}\right| \leq C\left(\left\|e_{h}^{n-1}\right\|_{1}^{2}+\left\|e_{h}^{n}\right\|_{1}^{2}+h^{2}\left|u^{n-1}\right|_{2}^{2}\right)
$$

From the Lipschitz property of $g(\mathbf{x}, u)$ in condition $\left(C_{2}\right)$, making use of triangle inequality, important inequality and Lemma 4 , we have

$$
\left|T_{4}\right| \leq C\left(h^{4}\left\|u^{n-1}\right\|_{2}^{2}+\left\|e_{h}^{n-1}\right\|_{0}^{2}+\left\|e_{h}^{n}\right\|_{0}^{2}\right),
$$

Combining (34),(35) with (36), we know that

$$
\left|T_{3}\right| \leq C\left(\left\|e_{h}^{n-1}\right\|_{1}^{2}+\left\|e_{h}^{n}\right\|_{1}^{2}+h^{2}\left\|u^{n-1}\right\|_{2}^{2}+h^{4}\left\|u^{n-1}\right\|_{1}^{2}+h^{2}\right)
$$

Combining (31), (32), (33) with (34) and applying Sobolev space embedding theory, we know that the RHS of (26) satisfies

$$
\begin{aligned}
R H S & \leq C\left(\left\|e_{h}^{n-1}\right\|_{0}^{2}+\left\|e_{h}^{n}\right\|_{0}^{2}+(\Delta t)^{2}\left\|u_{t t}^{n-1}\right\|_{0}^{2}\right. \\
& \left.+h^{2}\left(h^{2}\left|u_{t}^{n-1}\right|_{2}^{2}+\left\|u^{n-1}\right\|_{2}^{2}+1\right)\right) .
\end{aligned}
$$

From (30) and (35), using inverse estimate we know

$$
\begin{aligned}
& \frac{1}{2 \Delta t}\left[\left(\left\|e_{h}^{n}\right\|_{0}^{2}-\frac{\Delta t}{2}\left\|e_{h}^{n}\right\|_{1}^{2}\right)-\left(\left\|e_{h}^{n-1}\right\|_{0}^{2}-\frac{\Delta t}{2}\left\|e_{h}^{n-1}\right\|_{1}^{2}\right)\right] \\
& +\frac{1}{4} \mu\left\|e_{h}^{n}+e_{h}^{n-1}\right\|_{1}^{2} \leq C\left(\left\|e_{h}^{n-1}\right\|_{0}^{2}+\left\|e_{h}^{n}\right\|_{0}^{2}+(\Delta t)^{2}\left\|u_{t t}^{n-1}\right\|_{0}^{2}\right. \\
& \left.+h^{2}\left(h^{2}\left|u_{t}^{n-1}\right|_{2}^{2}+\left\|u^{n-1}\right\|_{2}^{2}+1\right)\right)
\end{aligned}
$$

Further, we get that

$$
\begin{aligned}
& {\left[\left(\left\|e_{h}^{n}\right\|_{0}^{2}-\frac{\Delta t}{2}\left\|e_{h}^{n}\right\|_{1}^{2}\right)-\left(\left\|e_{h}^{n-1}\right\|_{0}^{2}-\frac{\Delta t}{2}\left\|e_{h}^{n-1}\right\|_{1}^{2}\right)\right]} \\
& +\frac{\Delta t}{2} \mu\left\|e_{h}^{n}+e_{h}^{n-1}\right\|_{1}^{2} \leq C \Delta t\left(\left\|e_{h}^{n-1}\right\|_{0}^{2}+\left\|e_{h}^{n}\right\|_{0}^{2}\right. \\
& \left.+(\Delta t)^{2}\left\|u_{t t}^{n-1}\right\|_{0}^{2}+h^{2}\left(h^{2}\left\|u_{t}^{n-1}\right\|_{2}^{2}+\left\|u^{n-1}\right\|_{2}^{2}+1\right)\right)
\end{aligned}
$$

Summing from 1 to $N$ with respect to $n$ in the above inequality, we can obtain that

$$
\begin{aligned}
& {\left[\left(\left\|e_{h}^{N}\right\|_{0}^{2}-\frac{\Delta t}{2}\left\|e_{h}^{N}\right\|_{1}^{2}\right)-\left(\left\|e_{h}^{N-1}\right\|_{0}^{2}-\frac{\Delta t}{2}\left\|e_{h}^{N-1}\right\|_{1}^{2}\right)\right]} \\
& +\frac{\Delta t}{2} \mu \sum_{n=1}^{N}\left\|e_{h}^{n}+e_{h}^{n-1}\right\|_{1}^{2} \leq C \Delta t \sum_{n=1}^{N}\left(\left\|e_{h}^{n-1}\right\|_{0}^{2}+\left\|e_{h}^{n}\right\|_{0}^{2}\right) \\
& +C \Delta t \sum_{n=1}^{N}(\Delta t)^{2}\left\|u_{t t}^{n-1}\right\|_{0}^{2}+C \Delta t \sum_{n=1}^{N} h^{2}\left(h^{2}\left\|u_{t}^{n-1}\right\|_{2}^{2}+\left\|u^{n-1}\right\|_{2}^{2}+1\right)
\end{aligned}
$$

Noting the equivalence of $\|\cdot\| \|_{0}$ and $\|\cdot\| \|_{1}$ with $\|\cdot\|_{0}$ 
and \|\|$_{1}$, respectively. Using the inverse estimate, we have that there exist three positive constants $\sigma_{0}, \sigma_{1}, \sigma_{2}$ such that

$$
\sigma_{0}\|\cdot\|_{0} \leq\|\| \cdot\left\|_{0}^{2},\right\| \cdot\|\cdot\|_{1}^{2} \leq \sigma_{2}\|\cdot\|_{1}^{2} \leq \sigma_{1} h^{-2}\|\cdot\|_{0}^{2} .
$$

Further, (37) may be rewritten as

$$
\begin{aligned}
& \left(\sigma_{0}-\Delta t 2 \sigma_{1} h^{-2}\right)\left\|e_{h}^{N}\right\|_{0}^{2}+\Delta t 2 \sigma_{2} \sum_{n=0}^{N}\left\|e_{h}^{n}\right\|_{1}^{2} \\
& \leq C \Delta t \sum_{n=0}^{N}\left\|e_{h}^{n}\right\|_{0}^{2}+C \Delta t \sum_{n=0}^{N}(\Delta t)^{2}\left\|u_{t t}^{n-1}\right\|_{0}^{2} \\
& +C \Delta t \sum_{n=0}^{N} h^{2}\left(h^{2}\left|u_{t}^{n-1}\right|_{2}^{2}+\left\|u^{n-1}\right\|_{2}^{2}+1\right)
\end{aligned}
$$

Choosing $\Delta t, h$ in such way that

$$
\varepsilon_{0}=\sigma_{0}-\Delta t 2 \sigma_{1} h^{-2}>0,
$$

further, (38) can be rewritten as

$$
\begin{aligned}
& \left\|e_{h}^{N}\right\|_{0}^{2}+\eta \Delta t \sum_{n=0}^{N}\left\|e_{h}^{n}\right\|_{1}^{2} \\
& \leq C \Delta t \sum_{n=0}^{N}\left\|e_{h}^{n}\right\|_{0}^{2}+C \Delta t \sum_{n=0}^{N}(\Delta t)^{2}\left\|u_{t t}^{n-1}\right\|_{0}^{2} \\
& \quad+C \Delta t \sum_{n=0}^{N} h^{2}\left(h^{2}\left|u_{t}^{n-1}\right|_{2}^{2}+\left\|u^{n-1}\right\|_{2}^{2}+1\right),
\end{aligned}
$$

where $\eta=12 \varepsilon_{0}$. Using discrete Gronwall's lemma, we know that

$$
\begin{aligned}
& \left\|e_{h}^{N}\right\|_{0}^{2}+\eta \Delta t \sum_{n=0}^{N}\left\|e_{h}^{n}\right\|_{1}^{2} \\
& \leq C \Delta t \sum_{n=0}^{N}\left((\Delta t)^{2}\left\|u_{t t}^{n-1}\right\|_{0}^{2}+h^{2}\left(h^{2}\left|u_{t}^{n-1}\right|_{2}^{2}+\left\|u^{n-1}\right\|_{2}^{2}+1\right)\right) .
\end{aligned}
$$

Noting that $N \Delta t \leq T$, combining finite element space interpolation theory, we can obtain the resulting error estimates to the approximate solution as follows.

$$
\begin{aligned}
& \left\|u-u_{h}\right\|_{\bar{L}_{\infty}\left((0, T], H^{2}(\Omega)\right)}+\left\|u-u_{h}\right\|_{\bar{L}^{2}\left((0, T], H^{1}(\Omega)\right)} \\
& =O(h+\Delta t),
\end{aligned}
$$

where,

$$
\begin{aligned}
\|v\|_{\bar{L}_{\infty}((0, T], X)} & =\sup _{n \Delta t \leq T}\left\|v^{n}\right\|_{X},\|v\|_{\bar{L}^{2}((0, T], X)} \\
& =\sup _{N \Delta t \leq T}\left\{\eta \sum_{n=0}^{N}\left\|v^{n}\right\|_{X} \Delta t\right\}^{1 / 2} .
\end{aligned}
$$

Therefore we have the following theory.

Theorem 2. Suppose that the solution to the problem (1) is sufficiently smooth. When $h$ and $\Delta t$ are small enough and satisfy the relationship $\Delta t=O(h)$. The initial value $u_{h}^{0}$ is chosen as interpolation of $u_{0}$, then the Equation (44) holds.

\section{Conclusions}

In this paper, we continued our work [17] and presented a class of upwind FVEM based on tetrahedron partition for a three dimensional nonlinear convection diffusion equation, analyzed and derived error estimate in $L^{2}$ norm and $H^{1}$-norm for the method. In the ongoing work, we will discuss how to derive optimal error estimate in $L^{2}$-norm and how to code and present numerical results to demonstrate the performance.

\section{Acknowledgements}

The research was partially supported by the Scientific Research Award Fund for Excellent Middle-Aged and Young Scientists of Shandong Province (grant no. BS2009HZ015), and NSFC (grant no. 10801092).

\section{References}

[1] R. H. Li, Z. Y. Chen and W. Wu, "Generalized Difference Methods for Differential Equations: Numerical Analysis of Finite Volume Methods," Marcel Dekker, New York, 2000.

[2] Z. Q, Cai and S. F. McCormick, "On the Accuracy of the Finite Volume Element Method for Diffusion Equations on Composite Grids," SIAM Journal on Numerical Analysis, Vol. 27, 1990, pp. 635-655, 1990.

[3] Z. Q. Cai, J. Mandel and S. F. McCormick, "The Finite Volume Element Method for Diffusion Equations on General Triangulations," SIAM Journal on Numerical Analysis, Vol. 28, No. 2, 1991, pp. 392-402. doi: $10.1137 / 0728022$

[4] R. E. Bank and D. J. Rose, "Some Error Estimates for the Box Method," SIAM Journal on Numerical Analysis, Vol. 24, No. 4, 1987, pp. 777-787. doi:10.1137/0724050

[5] V. Patankar, "Numerical Heat Transfer and Fluid Flow," McGraw-Hill, New York, 1980.

[6] J. Douglas Jr. and T. F. Russell, "Numerical Methods for Convection-Dominated Diffusion Problems Based on Combining the Method of Characteristics with Finite Element or Finite Difference Procedures," SIAM Journal on $\mathrm{Nu}$ merical Analysis, Vol. 19, No. 5, 1982, pp. 871-885. doi: $10.1137 / 0719063$

[7] D. B. Spalding, "A Novel Finite Difference Formulation for Differential Equations Involving Both First and Second Derivatives," International Journal for Numerical Methods in Engineering, Vol. 4, No. 4, 1973, pp. 551-559. doi:10.1002/nme.1620040409

[8] K. Baba and M. Tabata, "On a Conservative Upwind Finite Element Scheme for Convective Diffusion Equations," RAIRO Analyse Numériqe, Vol. 15, No. 1, 1981, pp. 3-25.

[9] M. Tabata, "Uniform Convergence of the Upwind Finite Element Approximation for Semi-Linear Parabolic Problems," Journal of Mathematics of Kyoto University, Vol. 
18, No. 2, 1978, pp. 307-351.

[10] M. Tabata, "A Finite Element Approximation Corresponding to the Upwind Finite Differencing," Memoirs of Numerical Mathematics, Vol. 4, 1977, pp. 47-63.

[11] M. Tabata, "Conservative Upwind Finite Element Approximation and Its Applications, Analytical and Numerical Approaches to Asymptopic Problem in Analysis," NorthHolland, Amsterdam, 1981, pp. 369-387.

[12] Y. R. Yuan, "The Upwind Finite Difference Fractional Steps Methods for Two-phase Compressible Flow in Porous Media," Numerical Methods for Partial Differential Equations, Vol. 19, No. 1, 2003, pp. 67-88. doi:10.1002/num.10036

[13] D. Liang, "A Kind of Upwind Schemes for Convection Diffusion Equations," Mathematical Numerical Sinica, Vol. 2, 1991, pp. 133-141.

[14] Y. H. Li and R. H. Li, "Generalized Difference Methods on Arbitrary Quadrilateral Netwoks," Journal of Computational Mathematics, Vol. 17, No. 6, 1999, pp. 653-672.

[15] M. Feistauer, J. Felcman and M. Lukáčová-Medvid'ová, "On the Convergence of a Combined Finite Volume-Finite Element Method for Nonlinear Convection-Diffusion Problems," Numerical Methods for Partial Differential Equa- tions, Vol. 13, No. 2, 1997, pp. 163-190.

doi:10.1002/(SICI)1098-2426(199703)13:2<163::AID-N $\mathrm{UM} 3>3.0 . \mathrm{CO} ; 2-\mathrm{N}$

[16] M. Feistauer, J. Slavik and P. Stupka, "On the Convergence of a Combined Finite Volume-Finite Element Method for Nonlinear Convection-Diffusion Problems," Numerical Methods for Partial Differential Equations, Vol. 15, No. 2, 1999, pp. 215-235. doi:10.1002/(SICI)1098-2426(199903)15:2<215::AID-N UM6>3.0.CO;2-1

[17] F. Z. Gao and Y. R. Yuan, "An upwind Finite Volume Element Method Based on Quadrilateral Meshes for Nonlinear Convection-Diffusion Problems," Numerical Methods for Partial Differential Equations, Vol. 25, No. 5, 2009, pp. 1067-1085. doi:10.1002/num.20387

[18] P. G. Ciarlet, "The Finite Element Method for Elliptic Problems," North-Holland, Amsterdam, 1978.

[19] R. A. Adams, "Sobolev Spaces," Academic Press, New York, 1975.

[20] S. C. Brenner and L. R. Scott, "The Mathematics Theory of Finite Element Methods," Springer-Verlag, New York, 1994. 